Pacific Journal of Mathematics

REGULARITY OF CAPILLARY SURFACES OVER DOMAINS
WITH CORNERS: BORDERLINE CASE

LUEN-FAI TAM 


\title{
REGULARITY OF CAPILLARY SURFACES OVER DOMAINS WITH CORNERS: BORDERLINE CASE
}

\section{LUEN-FAI TAM}

\begin{abstract}
Consider the solutions of capillary surface equation with contact angle boundary condition over domains with corners. It is known that if the corner angle $2 \alpha$ satisfies $0<2 \alpha<\pi$ and $\alpha+\gamma>\pi / 2$ where $0<\gamma \leq \pi / 2$ is the contact angle, then solutions are regular. It is also known that no regularity holds in case $\alpha+\gamma<\pi / 2$. In this paper we show that solutions are still regular for the borderline case $\alpha+\gamma=\pi / 2$ at the corner.
\end{abstract}

It was proved by Concus and Finn in [1] that the behavior of a capillary surface near a corner over a wedge can change discontinuously. They proved that if the contact angle is $\gamma>0$ and the interior angle at the corner is $2 \alpha$, then all solutions for which $\alpha+\gamma \geq \pi / 2$ are bounded near the corner, while all solutions are unbounded if $\alpha+\gamma<\pi / 2$. Later in [9], Simon went further and investigated the regularity near the corner.

Let $\Omega$ be a domain contained in $B_{R}=\left\{x \in \mathbf{R}^{2}|| x \mid<R\right\}$ for some $R>0$, such that $\partial \Omega$ consists of a circular arc of $\partial B_{R}$ and two smooth Jordan arcs intersecting at the origin. Each arc makes an angle $\alpha$ with the positive $x^{1}$-axis, so that the interior angle at the origin is $2 \alpha$. See Figure 1 . Let $u$ be a bounded function satisfying

$$
\begin{cases}\operatorname{div} T u=H(x, u(x)) & \text { in } \Omega \\ T u=\frac{D u}{\sqrt{1+|D u|^{2}}} & \\ T u \cdot \nu=\cos \gamma & \text { on } \Gamma=(\partial \Omega-\{0\}) \cap B_{R}\end{cases}
$$

where $H(x, t)$ is a locally bounded function in $\bar{\Omega} \times \mathbf{R}, \pi / 2>\gamma>0$ is a constant angle and $\nu$ is the unit outward normal of $\Gamma$. If $u$ is smooth in $(\bar{\Omega}-\{0\})$ and if $\pi / 2>\alpha>\pi / 2-\gamma$, then Simon [9] proved that $u$ actually extends to be a $C^{1}$ function in $\bar{\Omega}$. It is known that no regularity holds if $\alpha+\gamma<\pi / 2$. Our aim is to examine the borderline case $\alpha+\gamma=$ $\pi / 2$. In this case, one cannot expect $D u$ to be continuous or even bounded in $\bar{\Omega}$, as one can easily construct counterexamples. Note also that 


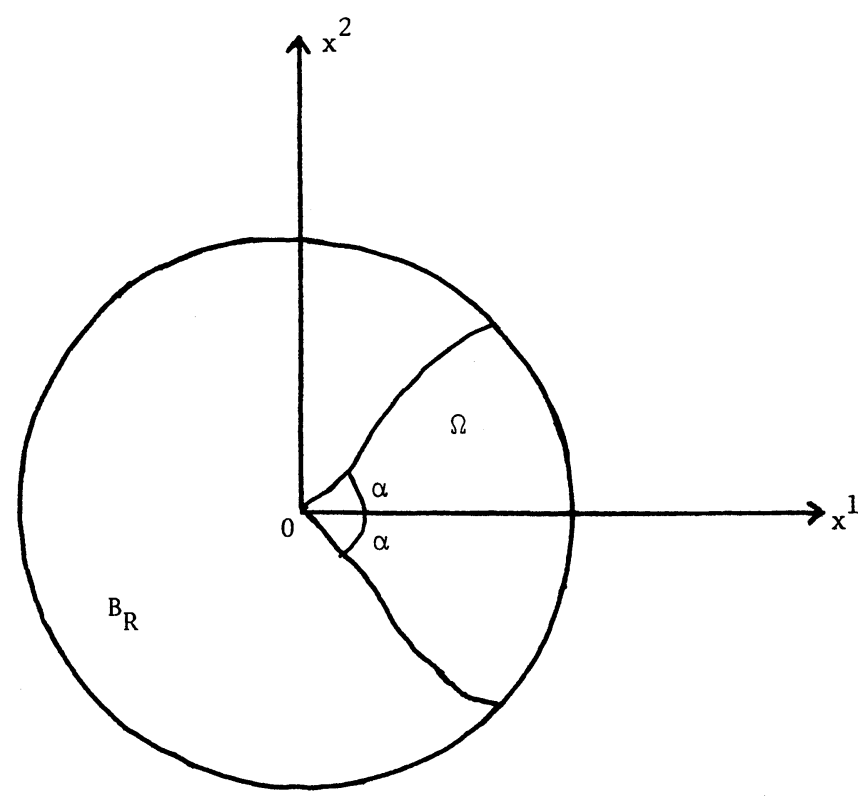

FIGURE 1

if $2 \alpha>\pi$, then there are examples which show that $u$ may be discontinuous at the corner, see [5]. In this paper we want to prove the following theorem:

THEOREM. Let $u \in C^{2}(\bar{\Omega}-\{0\}) \cap L^{\infty}(\Omega)$ be a solution of $(0.1)$. If $\alpha+\gamma=\pi / 2$, then $u$ and $\left(T u,-1 / \sqrt{1+|D u|^{2}}\right)$ extend to be continuous functions in $\bar{\Omega}$ with values in $\mathbf{R}$ and $\mathbf{R}^{3}$ respectively.

Since $H(x, t)$ is locally bounded in $\bar{\Omega} \times \mathbf{R}$ and $u \in L^{\infty}(\Omega)$, so we may assume that $u$ satisfies:

$$
\begin{cases}\operatorname{div} T u=H & \text { in } \Omega \\ T u \cdot \nu=\cos \gamma & \text { on } \Gamma\end{cases}
$$

for some bounded continuous function $H=H(x)$ in $\Omega$.

1. Continuity of $u$ at the corner. Let $(0, a) \in \mathbf{R}^{2} \times \mathbf{R}=\mathbf{R}^{3}$ be any point lying in the closure of the graph of $u$ over $\Omega$.

Define $v(x)=u(x)-a$.

THEOREM 1.1. Under the above assumptions, we have

$$
\lim _{\substack{x \rightarrow 0 \\ x \in \Omega}} \frac{v(x)}{x^{1}}=-\infty \quad \text { where } x=\left(x^{1}, x^{2}\right) \in \mathbf{R}^{2} .
$$


Note that if $x$ is close enough to the origin, we have $x^{1}>0$. Therefore without loss of generality, we may assume that $x^{1}>0$ for all $x \in \Omega$.

Proof. Suppose that (1.1) is not true, then there exists a real number $M$ and a sequence of points $x_{j} \in \Omega$ such that $\lim _{j \rightarrow \infty} x_{j}=0$ and

$$
\frac{v\left(x_{j}\right)}{x_{j}^{1}} \geq M
$$

We want to get a contradiction from this. For this purpose we need several lemmas.

With minor modifications, the proofs of Lemma 1.2-1.6 in the following can be found in the literature. So we shall not prove them, but only give the references. We state them here for the convenience of the reader.

Let $\varepsilon_{j}=x_{j}^{1}$, then $\lim _{j \rightarrow \infty} \varepsilon_{j}=0$. Define $v_{j}(x)=v\left(\varepsilon_{j} x\right) / \varepsilon_{j}$. Then $v_{j}(x)$ satisfies:

$$
\begin{cases}\operatorname{div} T v_{j}=\varepsilon_{j} H & \text { in } \Omega_{j}=\left\{x \in \mathbf{R}^{2} \mid \varepsilon_{j} x \in \Omega\right\}, \\ T v_{j} \cdot \nu_{j}=\cos \gamma & \text { on } \Gamma_{j}=\left\{x \in \mathbf{R}^{2} \mid \varepsilon_{j} x \in \Gamma\right\},\end{cases}
$$

where $\nu_{j}$ is the unit outward normal of $\Gamma_{j}$. Notice that $v_{j} \in C^{2}\left(\bar{\Omega}_{j}-\{0\}\right)$ $\cap L^{\infty}\left(\Omega_{j}\right)$ for all $j$.

Let $\Omega_{\infty}=\lim _{j \rightarrow \infty} \Omega_{j}=\left\{x \in \mathbf{R}^{2}|| x^{2} \mid<(\tan \alpha) x^{1}\right\}$.

As shown in [9] (see also [3] and [10]), noting that $\varepsilon_{j} H$ tend to zero everywhere in $\Omega_{\infty}$, and $\varepsilon_{j} H$ are uniformly bounded, using the terminology in [3] we have:

LEMMA 1.2. We can find a subsequence of $v_{j}$ which converges locally to a generalized solution $v_{\infty}$ in $\Omega_{\infty}$ of

$$
\mathscr{F}(w) \equiv \int_{\Omega_{\infty}} \sqrt{1+|D w|^{2}}-\cos \gamma \int_{\partial \Omega_{\infty}} w d H_{1}
$$

where $H_{k}$ is the $k$-dimensional Hausdorff measure in $\mathbf{R}^{n}, k \leq n$. That is to say, if $V_{\infty}=\left\{(x, t) \in \Omega_{\infty} \times \mathbf{R} \mid t<v_{\infty}(x)\right\}$ is the subgraph of $v_{\infty}$, then for any compact set $K \subset \mathbf{R}^{3}$, and for any Caccioppoli set (set of locally finite perimeter) $E$, such that $\operatorname{spt}\left(\varphi_{V_{\infty}}-\varphi_{E}\right) \subset K$, we have

$$
F_{K}\left(V_{\infty}\right) \leq F_{K}(E)
$$

where

$$
F_{K}(W) \equiv \int_{\left(\Omega_{\infty}^{\prime} \times \mathbf{R}\right) \cap K}\left|D \varphi_{W}\right|-\cos \gamma \int_{\left(\partial \Omega_{\infty} \times \mathbf{R}\right) \cap K} \varphi_{W} d H_{2}
$$


and where $\varphi_{W}$ denotes the characteristic function of $W$.

A sequence of functions $f_{j}$ is said to converge locally to a function $f$ in a domain $D$, if the characteristic functions of the subgraphs of $f_{j}$ converge almost everywhere to the characteristic function of the subgraph of $f$ in $D \times \mathbf{R}$.

Note that $v_{\infty}$ may take the value $\infty$ or $-\infty$.

Define

$$
\begin{gathered}
P=\left\{x \in \Omega_{\infty} \mid v_{\infty}(x)=\infty\right\} \\
N=\left\{x \in \Omega_{\infty} \mid v_{\infty}(x)=-\infty\right\} .
\end{gathered}
$$

As in [3] (see also $[9,10])$, we know that $P$ minimizes

$$
G(A) \equiv \int_{\Omega_{\infty}}\left|D \varphi_{A}\right|-\cos \gamma \int_{\partial \Omega_{\infty} \cap K} \varphi_{A} d H_{1}
$$

for Caccioppoli set $A \subset \Omega_{\infty}$. That is, for any compact set $K \subset \mathbf{R}^{2}$, and any Caccioppoli set with $\operatorname{spt}\left(\varphi_{A}-\varphi_{P}\right) \subset K$, we have

$$
G_{K}(P) \equiv \int_{\Omega_{\infty} \cap K}\left|D \varphi_{P}\right|-\cos \gamma \int_{\partial \Omega_{\infty} \cap K} \varphi_{P} d H_{1} \leq G_{K}(A)
$$

Similarly, $N$ minimizes

$$
G^{\prime}(A) \equiv \int_{\Omega_{\infty}}\left|D \varphi_{A}\right|+\cos \gamma \int_{\partial \Omega_{\infty}} \varphi_{A} d H_{1}
$$

We want to know the structure of $P$ and $N$, and we have:

LemMA 1.3. If $L \subset \Omega_{\infty}$ minimizes $G(A)$ defined in (1.9), then $L$ equals to $\Omega_{\infty}$, $\varnothing$ or some $\triangle O A B$ bounded by $\partial \Omega_{\infty}$ and $x^{1}=$ a for some $a>0$. (See Figure 2.)

The proof of the lemma is similar to the proof of Theorem 2.4 for the case $\alpha+\gamma>\pi / 2$ in [10]. In that case, the conclusion is that $L=\Omega_{\infty}$ or $\varnothing$. In our case, it is possible to have $L=\triangle O A B$ described in the lemma because $2 \alpha+2 \gamma=\pi$. We shall omit the proof. Similarly we have:

LeMMA 1.4. If $L$ minimizes $G^{\prime}(A)$ defined by (1.11), then $L$ equals to $\Omega_{\infty}, \varnothing$ or $\Omega_{\infty}-\triangle O A B$ for some $\triangle O A B$ described in Lemma 1.3.

Since $P$ minimizes $G(A)$ and $N$ minimizes $G^{\prime}(A)$, we conclude that

(1.12) $P=\Omega_{\infty}, \varnothing$ or $\triangle O A B$ which is bounded by $\partial \Omega_{\infty}$ and $x^{1}=a$ for some $a>0$. 
REGULARITY OF CAPILLARY SURFACES

473

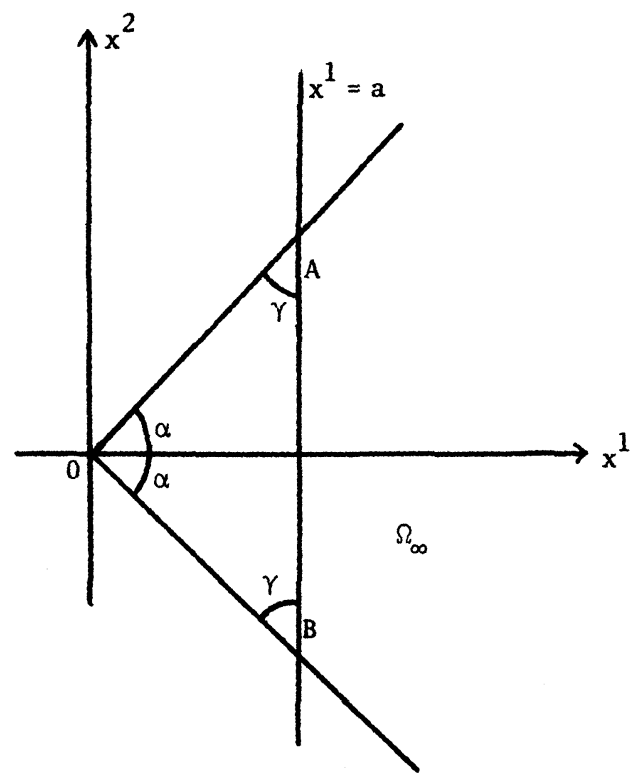

FIGURE 2

(1.13) $N=\Omega_{\infty}$, $\varnothing$ or $\Omega_{\infty}-\triangle O A^{\prime} B^{\prime}$ for some $\triangle O A^{\prime} B^{\prime}$ which is bounded by $\partial \Omega_{\infty}$ and $x^{1}=a^{\prime}$ for some $a^{\prime}>0$.

It is not hard to see from the proof of Lemma 3.1 in [11] that the following estimates are true. (See also [3].) Let $V_{j}$ be the subgraph of $v_{j}$.

LEMma 1.5. There exists $r_{0}>0, C>0$ not depending on $j$ such that for all $t \in \mathbf{R}$, the following is true:

if $\left|V_{j, r}^{\prime}(0, t)\right|>0$ for all $r>0$ then $\left|V_{j, r}^{\prime}(0, t)\right| \geq C r^{3}$ for all $0<r \leq r_{0}$, where $C_{r}\left(x_{0}, t_{0}\right)=\left\{(x, t) \in \mathbf{R}^{3}|| x-x_{0} \mid\right.$ $<r$ and $\left.\left|t-t_{0}\right|<r\right\}$ and $V_{j, r}^{\prime}(0, t)=C_{r}(0, t)-V_{j}$.

LEMMA 1.6. For any $0<\tau_{1}<\tau_{2}<\infty$, there exist positive integer $j_{0}$ and positive numbers $r_{1}$ and $C_{1}$ such that for all $j \geq j_{0}$ and $(x, t) \in \Omega_{j} \cap$ $\left\{x \in \mathbf{R}^{2} \mid \tau_{1} \leq x^{1} \leq \tau_{2}\right\}$, the following are true:

if $\left|V_{j, r}(x, t)\right|>0$ for all $r>0$, then $\left|V_{j, r}(x, t)\right| \geq C_{1} r^{3}$, for all $0<r \leq r_{1}$;

if $V_{j, r}^{\prime}(x, t)>0$ for all $r>0$, then $\left|V_{j, r}^{\prime}(x, t)\right| \geq C_{1} r^{3}$ for all $0<r \leq r_{1}$,

where $V_{j, r}(x, t)=C_{r}(x, t) \cap V_{j}$ and $V_{j, r}^{\prime}(x, t)=C_{r}(x, t)-V_{j}$. 
Notice that even though we do not have a similar result as (1.15) at the corner (because of the fact that $\alpha+\gamma=\pi / 2$ ), we still have (1.14) since $\cos \gamma>0$, as one can see from the proof of Lemma 3.1 in [11].

Using the above lemmas, we can prove:

LEMMA 1.7. $P=\left\{x \in \Omega_{\infty} \mid v_{\infty}(x)=\infty\right\}$ is empty.

Proof. If $P \neq \varnothing$, then by Lemma 1.3, $P=\Omega_{\infty}$ or some $\triangle O A B$ which is bounded by $\partial \Omega_{\infty}$ and $x^{1}=a$ for some $a>0$. In any case, there is $\bar{r}>0$ such that

$$
\left|V_{\infty, r}^{\prime}(0,0)\right|=\left|C_{r}(0,0)-V_{\infty}\right|=0 \text { for all } 0<r \leq \bar{r} .
$$

By Lemma 1.5 and the fact that $(0,0) \in \mathbf{R}^{3}$ lies in the closure of the graph of $v_{j}$ and that $v_{j}$ is regular in $\bar{\Omega}_{j}-\{0\}$, we have:

$$
\begin{aligned}
& \left|V_{j, r}^{\prime}(0,0)\right|>0 \text { for all } r>0, \text { and so } \\
& \left|V_{j, r}^{\prime}(0,0)\right| \geq C r^{3} \quad \text { for all } 0<r \leq r_{0} .
\end{aligned}
$$

In particular, if we take $r=\min \left(\bar{r}, r_{0}\right)>0$, then

$$
\left|V_{j, r}^{\prime}(0,0)\right| \geq C r^{3} \text {. }
$$

Let $j \rightarrow \infty$, noting that $\varphi_{V_{j}}$ converges to $\varphi_{V_{\infty}}$ almost everywhere in $\Omega_{\infty} \times \mathbf{R}$, we have

$$
\left|V_{\infty, r}^{\prime}(0,0)\right| \geq C r^{3}>0
$$

This contradicts (1.17). Therefore $P$ must be empty and the lemma is proved.

LEMMA 1.8. If $N=\left\{x \in \Omega_{\infty} \mid v_{\infty}=-\infty\right\}$, then $N=\Omega_{\infty}$.

Proof. By (1.13) and Lemma 1.7, if $N \neq \Omega_{\infty}$, then there exists $\tau>0$ such that $v_{\infty}$ is finite almost everywhere in $\left\{x \in \Omega_{\infty} \mid 0<x^{1}<\tau\right\}$. We claim that there is a positive integer $j_{0}$ such that

$$
\sup _{j \geq j_{0}} \sup _{\substack{x \in \Omega_{j} \\ \tau / 4<x^{1}<3 \tau / 4}}\left|v_{j}(x)\right|<\infty .
$$

Let $j_{0}, r_{1}$, and $C_{1}$ be the constants in Lemma 1.6 corresponding to $\tau_{1}=\tau / 4$, and $\tau_{2}=3 \tau / 4$.

Since each $v_{j}$ is bounded in $\Omega_{j}$, if (1.18) is not true, then we can find a subsequence of $v_{j}$, which we also call $v_{j}$, and $\bar{x}_{j} \in \Omega_{j}, \tau / 4<\bar{x}_{j}<3 \tau / 4$, such that

$$
\lim _{j \rightarrow \infty}\left|v_{j}\left(\bar{x}_{j}\right)\right|=\infty
$$


Passing to a subsequence if necessary, we may assume that $\lim _{j \rightarrow \infty} \bar{x}_{j}$ $=z=\left(z^{1}, z^{2}\right)$ which is in $\bar{\Omega}_{\infty}$, with $\tau / 4 \leq z^{1} \leq 3 \tau / 4$, and such that

$$
\begin{aligned}
& \lim _{j \rightarrow \infty} v_{j}\left(\bar{x}_{j}\right)=\infty, \text { or } \\
& \lim _{j \rightarrow \infty} v_{j}\left(\bar{x}_{j}\right)=-\infty .
\end{aligned}
$$

Suppose that $\lim _{j \rightarrow \infty} v_{j}\left(\bar{x}_{j}\right)=\infty$. Then for any $t>0$, if $j$ is large enough, we have

$$
\left|V_{j, r}\left(\bar{x}_{j}, t\right)\right|>0 \text { for all } r>0 \text {. }
$$

Hence by (1.15), if $j$ is large enough, we have

$$
\left|V_{j, r}\left(\bar{x}_{j}, t\right)\right| \geq C_{1} r^{3} \quad \text { for all } 0<r \leq r_{1} .
$$

Let $j \rightarrow \infty$, we get

$$
\left|V_{\infty, r}(z, t)\right| \geq C_{1} r^{3} \quad \text { for all } 0<r \leq r_{1} .
$$

Since $t$ can be arbitrarily large, this contradicts the fact that $P=\varnothing$.

Suppose that $\lim _{j \rightarrow \infty} v_{j}\left(\bar{x}_{j}\right)=-\infty$, then for any $t<0$, if $j$ is large enough, we have

$$
\left|V_{j, r}^{\prime}\left(\bar{x}_{j}, t\right)\right|>0 \text { for all } r>0 \text {. }
$$

By (1.16), we have

$$
\left|V_{j, r}^{\prime}\left(\bar{x}_{j}, t\right)\right| \geq C_{1} r^{3} \quad \text { for all } 0<r \leq r_{1} .
$$

Take $\bar{r}=\min \left(\frac{1}{4} \tau, r_{1}\right)>0$ and let $j \rightarrow \infty$, we get

$$
\left|V_{\infty, \bar{r}}^{\prime}(z, t)\right| \geq C_{1} \bar{r}^{3} \quad \text { for all } t<0 \text {. }
$$

Since $t$ can be arbitrarily small, this contradicts the fact that $v_{\infty}$ is finite almost everywhere in $\left\{x \in \Omega_{\infty} \mid 0<x^{1}<\tau\right\}$.

In any case, we have a contradiction. Therefore (1.18) is true.

By Theorem 3 in [7], $v_{\infty}$ is regular in $D=\left\{x \in \Omega_{\infty} \mid \tau / 4<x^{1}<\right.$ $3 r / 4\}$ after modification by a set of measure zero. By the results of [6], we have

$$
\left\{\begin{array}{l}
\lim _{j \rightarrow \infty} v_{j}(x)=v(x) \\
\lim _{j \rightarrow \infty} D v_{j}(x)=D v(x)
\end{array}\right.
$$

for $x \in D$. Integrating $\operatorname{div} T v_{j}=\varepsilon_{j} H$ over $D_{j}=\left\{x \in \Omega_{j} \mid 0<x^{1}<\tau / 2\right\}$, using (1.3) and let $\eta=(-1,0,0)$, we have, for $j$ large enough:

$$
\int_{\Gamma_{j} \cap\left\{0<x^{1}<\tau / 2\right\}} T v_{j} \cdot v_{j} d H_{1}=\int_{D_{j}} \varepsilon_{j} H d x+\int_{D_{j} \cap\left\{x^{1}=\tau / 2\right\}} T v_{j} \cdot \eta d H_{1} .
$$



we get

Since $T v_{j} \cdot \nu_{j}=\cos \gamma$ on $\Gamma_{j}$, and $\lim _{j \rightarrow \infty} \varepsilon_{j} H=0$, if we let $j \rightarrow \infty$,

$$
\cos \gamma \cdot H_{1}\left(\partial \Omega_{\infty} \cap\left\{0<x^{1}<\frac{\tau}{2}\right\}\right)=\int_{D \cap\left\{x^{1}=\tau / 2\right\}} T v_{\infty} \cdot \eta d H_{1} .
$$

But

$$
\cos \gamma \cdot H_{1}\left(\partial \Omega_{\infty} \cap\left\{0<x^{1}<\frac{\tau}{2}\right\}\right)=H_{1}\left(D \cap\left\{x^{1}=\frac{\tau}{2}\right\}\right) .
$$

Since $\left|T v_{\infty} \cdot \eta\right| \leq 1$, we conclude that $T v_{\infty} \cdot \eta=1 H_{1}$-almost everywhere on $D \cap\left\{x^{1}=\tau / 2\right\}$. This contradicts the fact that $v_{\infty}$ is regular in $D$. Hence we must have $N=\Omega_{\infty}$.

REMARK. We may simplify the proof by using the fact that $V_{\infty}$ is a cone with vertex at the origin. But in the next section we shall use a similar argument, so we do it this way.

Conclusion of the proof of Theorem 1.1. Using the fact that $N=\Omega_{\infty}$ and using (1.15) and similar method of proof of (1.18), we can conclude that

$$
\lim _{j \rightarrow \infty} \sup _{\substack{x \in \Omega_{j} \\ 1 \leq x^{1} \leq 3 / 2}} v_{j}(x)=-\infty .
$$

In particular, we have

$$
\lim _{j \rightarrow \infty} \frac{v\left(x_{j}\right)}{x_{j}^{1}}=\lim _{j \rightarrow \infty} v_{j}\left(1, \frac{x_{j}^{2}}{x_{j}^{1}}\right)=-\infty .
$$

This contradicts (1.2), and the proof of Theorem 1.1 is complete.

Now we can prove the continuity of $u$.

THEOREM 1.9. $u$ extends to be a continuous function in $\bar{\Omega}$.

Proof. If this is not true, then there exist real numbers $b>a$, such that $(0, a)$ and $(0, b)$ are both in the closure of the graph of $u$. Let $v=u-a$. By Theorem 1.1, we have

$$
\lim _{\substack{x \rightarrow 0 \\ x \in \Omega}} \frac{v(x)}{x^{1}}=-\infty .
$$


In particular, there exists $r>0$, such that if $x \in \Omega$ and $|x|<r$, then $v(x) / x^{1}<0$. Therefore $u(x)<a$ for such $x$. Since $(0, b)$ also lies in the closure of the graph of $u$, we can always find $x \in \Omega$ with $0<|x|<r$ and $u(x)>a$. This leads to contradiction and the theorem follows.

2. Continuity of the normal. Let us proceed and examine the continuity of the normal of the graph of $u$ over $\Omega$. Since $u$ is continuous at the origin, by adding a constant to $u$, we may assume that $u(0)=0$. $u$ still satisfies (0.2). We want to prove:

$$
\lim _{\substack{x \rightarrow 0 \\ x \in \Omega}}\left(T u, \frac{-1}{\sqrt{1+|D u|^{2}}}\right)=(-1,0,0) .
$$

Since $u \in C^{2}(\bar{\Omega}-\{0\})$, it is sufficient to prove that for any sequence $x_{j} \in \Omega$, converging to 0 , we have

$$
\lim _{j \rightarrow \infty}\left(T u\left(x_{j}\right), \frac{-1}{\sqrt{1+\left|D u\left(x_{j}\right)\right|^{2}}}\right)=(-1,0,0) .
$$

First, we shall establish (2.1) for any sequence $x_{j}$ tending to the origin non-tangentially to $\partial \Omega$. More precisely, we assume that there is $\varepsilon$ with $0<\varepsilon<\tan \alpha$, such that $x_{j}=\left(x_{j}^{1}, x_{j}^{2}\right)$ lies between the straight lines $x^{2}= \pm(\tan \alpha-\varepsilon) x^{1}$.

THEOREM 2.1. Let $x_{j}=\left(x_{j}^{1}, x_{j}^{2}\right) \in \Omega$ be a sequence of points approaching the origin such that $\left|x_{j}^{2}\right|<(\tan \alpha-\varepsilon) x_{j}^{1}$ for all $j$ for some $\varepsilon$ with $0<\varepsilon<\tan \alpha$. Then (2.1) holds.

Proof. If we can prove that for any subsequence of $x_{j}$, we can find a subsequence of the subsequence such that (2.1) is true for that subsequence, then we are done.

Since every subsequence of $x_{j}$ also satisfies the assumptions of the theorem, so we may assume that the subsequence is $\left\{x_{j}\right\}$ itself.

Since $x_{j}^{1}>0$ for all $j$, if we set $\varepsilon_{j}=x_{j}^{1}$ and define

$$
u_{j}(x)=\frac{1}{\varepsilon_{j}} u\left(\varepsilon_{j} x\right)-\frac{1}{\varepsilon_{j}} u\left(x_{j}\right)
$$

then as in $\$ 1, u_{j}$ satisfies:

$$
\begin{cases}\operatorname{div} T u_{j}=\varepsilon_{j} H & \text { in } \Omega_{j} \\ T u_{j} \cdot \nu_{j}=\cos \gamma & \text { on } \Gamma_{j}\end{cases}
$$


Also if

$$
\bar{x}_{j}=\left(1, x_{j}^{2} / \varepsilon_{j}\right)=\left(1, x_{j}^{2} / x_{j}^{1}\right)
$$

then

$$
u_{j}\left(\bar{x}_{j}\right)=0
$$

We may also assume that

$$
\lim _{j \rightarrow \infty} \bar{x}_{j}=z=\left(1, z^{2}\right) \in \Omega_{\infty} \quad \text { with }\left|z^{2}\right| \leq \tan \alpha-\varepsilon .
$$

As in $\$ 1$, we can find a subsequence of $u_{j}$, which we also call $u_{j}$, converging locally to a generalized solution $u_{\infty}$ of $\mathscr{F}(w)$ defined by (1.4). Let

$$
P=\left\{x \in \Omega_{\infty} \mid u_{\infty}(x)=+\infty\right\}
$$

and

$$
N=\left\{x \in \Omega_{\infty} \mid u_{\infty}(x)=-\infty\right\} .
$$

As in $\S 1$, we know that $P=\Omega_{\infty}, \varnothing$ or some $\triangle O A B$ bounded by $\partial \Omega_{\infty}$ and $x^{1}=a$ for some $a>0$; and $N=\Omega_{\infty}, \varnothing$ or $\Omega_{\infty}-\triangle O A^{\prime} B^{\prime}$ for some $\triangle O A^{\prime} B^{\prime}$ bounded by $\partial \Omega_{\infty}$, and $x^{1}=a^{\prime}$ for some $a^{\prime}>0$.

Note that Lemma 1.6 is still true for the subgraph $U_{j}$ of $u_{j}$. That is to say for any $0<\tau_{1}<\tau_{2}<\infty$, there exist a positive integer $j_{0}$ and positive numbers $r_{1}$ and $C_{1}$ not depending on $j$ such that for $j \geq j_{0}$ and for any $(x, t) \in \bar{\Omega}_{j} \cap\left\{x \in R^{2} \mid \tau_{1}<x^{1}<\tau_{2}\right\},(1.15)$ and (1.16) are still true if we replace $V_{j}$ by $U_{j}$.

Suppose that $\Omega_{\infty}-(P \cup N) \neq \varnothing$, because of the structures of $P$ and $N$, there exist $0<a<b<\infty$ such that $u_{\infty}$ is finite almost everywhere in $\left\{x \in \Omega_{\infty} \mid a<x^{1}<b\right\}$. Using (1.15) and (1.16) as in the proof of Lemma 1.8, we shall arrive at a contradiction.

Hence we must have $\Omega_{\infty}=P \cup N$.

Let $U_{\infty}$ be the subgraph of $u_{\infty}$. Since $u_{j}\left(\bar{x}_{j}\right)=0$ so $\left(\bar{x}_{j}, 0\right)$ belongs to the boundary of $U_{j}$. Using (1.15), (1.16), the fact that $\lim _{j \rightarrow \infty} \bar{x}_{j}=z$, $u_{j} \in C^{2}(\bar{\Omega}-\{0\})$, and that $\varphi_{U}$ converge to $\varphi_{U_{\infty}}$ almost everywhere in $\Omega_{\infty} \times \mathbf{R}$, we have:

$$
\left|U_{\infty, r}(z, 0)\right| \geq C_{1} r^{3}, \text { and }\left|U_{\infty, r}^{\prime}(z, 0)\right| \geq C_{1} r^{3}
$$

for all $0<r \leq r_{1}$. Hence $P \neq \Omega_{\infty}$ and $N \neq \Omega_{\infty}$. Combining this with the fact that $\Omega_{\infty}=P \cup N$, we conclude that there is an $a>0$ such that if $O A B$ is the triangle bounded by $\partial \Omega_{\infty}$ and $x^{1}=a$, then $P=\triangle O A B$ and $N=\Omega_{\infty}-\triangle O A B$. So $U_{\infty}=\triangle O A B \times \mathbf{R}$. 
In fact, we must have $a=1$. Otherwise, as $z=\left(1, z^{2}\right), a<1$ will give a contradiction to the first inequality of (2.5), while $a>1$ will give a contradiction to the second inequality of (2.5).

The inward normal of $\partial U_{\infty}$ at $(z, 0) \in \mathbf{R}^{3}$ is $(-1,0,0)$, and the inward normal of $\partial U_{j}$ at $\left(\bar{x}_{j}, u\left(\bar{x}_{j}\right)\right)$ is $\left(T u_{j}\left(\bar{x}_{j}\right),-1 / \sqrt{1+\left|D u_{j}\left(\bar{x}_{j}\right)\right|^{2}}\right)$. Since $\lim _{j \rightarrow \infty}\left(\bar{x}_{j}, u_{j}\left(\bar{x}_{j}\right)\right)=(z, 0)$, so by Theorem 3 in [6], we have:

$$
\lim _{j \rightarrow \infty}\left(T u_{j}\left(\bar{x}_{j}\right), \frac{-1}{\sqrt{1+\left|D u_{j}\left(\bar{x}_{j}\right)\right|^{2}}}\right)=(-1,0,0) .
$$

From the definitions of $u_{j}$ and $\bar{x}_{j}$, we conclude that

$$
\lim _{j \rightarrow \infty}\left(T u\left(x_{j}\right), \frac{-1}{\sqrt{1+\left|D u\left(x_{j}\right)\right|^{2}}}\right)=(-1,0,0) .
$$

Finally, we consider the case when $x_{j}$ approaches the origin tangentially along $\partial \Omega_{\infty}$. We want to prove:

THEOREM 2.2. Under the above assumptions, (2.1) is still true, namely:

$$
\lim _{j \rightarrow \infty}\left(T u\left(x_{j}\right), \frac{-1}{\sqrt{1+\left|D u\left(x_{j}\right)\right|^{2}}}\right)=(-1,0,0) .
$$

Proof. As in Theorem 2.1, it is sufficient to prove that (2.1) is true for a subsequence of $x_{j}$.

Define $u_{j}$ and $\bar{x}_{j}$ as in Theorem 2.1. We also assume that $\lim _{j \rightarrow \infty} \bar{x}_{j}$ $=z=\left(1, z^{2}\right)$ which lies in $\bar{\Omega}_{\infty}$, with $z^{2}= \pm \tan \alpha$.

We can extract a subsequence of $u_{j}$, which we also denote by $u_{j}$, such that $u_{j}$ converges locally to a generalized solution of $\mathscr{F}(w)$ in $\Omega_{\infty}$.

Using similar method as in Theorem 2.1, we can prove that the subgraph $U_{\infty}$ of $u_{\infty}$ is $\triangle O A B \times \mathbf{R}$ for some $\triangle O A B$ bounded by $\partial \Omega_{\infty}$ and $x^{1}=1$. Up to this point, the proof is exactly the same as the proof in Theorem 2.1. However, in this case $z \in \partial \Omega_{\infty}$ and we cannot apply the results of [6]. So we need some modifications. Before we proceed further, let us prove the following lemma.

LEMMA 2.3. (a) For any $0<\tau_{1}<\tau_{2}<1$, we have

$$
\lim _{j \rightarrow \infty} \inf _{\substack{x \in \bar{\Omega}_{j} \\ \tau_{1}<x^{1}<\tau_{2}}} u_{j}(x)=\infty ; \text { and }
$$


(b) For any $1<\tau_{3}<\tau_{4}<\infty$, we have

$$
\lim _{j \rightarrow \infty} \sup _{\substack{x \in \bar{\Omega}_{j} \\ \tau_{3}<x^{1}<\tau_{4}}} u_{j}(x)=-\infty .
$$

Proof. We shall prove (a) only, because the proof of (b) is similar.

Suppose that (2.6) is not true. Since $u_{j} \in C^{2}\left(\bar{\Omega}_{j}-\{0\}\right)$, therefore we can find a real number $M$, a subsequence of $u_{j}$ (which we also call $u_{j}$ ) and a sequence of points $y_{j} \in \Omega_{j}, \tau_{1}<y_{j}^{1}<\tau_{2}$ such that

$$
u_{j}\left(y_{j}\right) \leq M \text {. }
$$

We may also assume that $\lim _{j \rightarrow \infty} y_{j}=y \in \bar{\Omega}_{\infty}$. Note that $\tau_{1} \leq y^{1} \leq \tau_{2}$. By (1.16) as before, we have

$$
\left|U_{j, r}^{\prime}\left(y_{j}, M\right)\right| \geq C_{1} r^{3}
$$

for all $0<r \leq r_{1}$ if $j$ is large enough, where $C_{1}$, and $r_{1}$ are positive constants not depending on $j$. Now let $j \rightarrow \infty$, we have

$$
\left|U_{\infty, r}^{\prime}(y, M)\right| \geq C_{1} r^{3} \quad \text { for all } 0<r \leq r_{1} .
$$

This contradicts the fact that $U_{\infty}=\triangle O A B \times \mathbf{R}$ and that $0<\tau_{1}<\tau_{2}<1$, bearing in mind the definition of $\triangle O A B$. The lemma is then proved.

We now continue our proof of Theorem 2.2. By Lemma 2.3, since $u_{j}$ is continuous in $\bar{\Omega}_{j}-\{0\}$, there exists $j_{0}$ such that for every $j \geq j_{0}$ we can find $y_{j} \in \partial \Omega_{j}$ with $u_{j}\left(y_{j}\right)=0$ and $\lim _{j \rightarrow \infty} y_{j}=z$.

Let $Y_{j}=\left(y_{j}, u_{j}\left(y_{j}\right)\right)=\left(y_{j}, 0\right) \in \mathbf{R}^{3}$. By the results of [12], there exist $r_{2}>0, C_{2}>0$ and $1>\alpha>0$ not depending on $j$ such that if $\eta_{j}(X)$ is the unit inward normal of $\partial U_{j}$ at the point $X \in \partial U_{j} \cap \Omega_{j}$ we have

$$
\left|\eta_{j}(X)-\eta_{j}(\bar{X})\right| \leq C_{2}|X-\bar{X}|^{\alpha}
$$

for any $X, \bar{X}$ belong to $\partial U_{j} \cap \Omega_{j}$ and $B_{r_{2}}\left(Y_{j}\right)=\left\{X \in \mathbf{R}^{3}|| X-Y_{j} \mid<r_{2}\right\}$.

For any $r_{2} / 2>r>0$, use Lemma 2.3 again, we can find $z_{j} \in \Omega_{j}$ and $\varepsilon$ with $\tan \alpha>\varepsilon>0$ not depending on $j$ such that if $j$ is large enough, we have

$$
\left\{\begin{array}{l}
\left|z_{j}^{2}\right|<(\tan \alpha-\varepsilon) z_{j}^{1} \\
u_{j}\left(z_{j}\right)=0 \\
\left|z_{j}-z\right|<r \\
\lim _{j \rightarrow \infty} z_{j}^{1}=1
\end{array}\right.
$$


Let $Z_{j}=\left(z_{j}, u_{j}\left(z_{j}\right)\right)=\left(z_{j}, 0\right), \quad Z=(z, 0)$ and $\bar{X}_{j}=\left(\bar{x}_{j}, u_{j}\left(\bar{x}_{j}\right)\right)=$ $\left(\bar{x}_{j}, 0\right)$.

Then $\lim _{j \rightarrow \infty} Y_{j}=Z=\lim _{j \rightarrow \infty} \bar{X}_{j}$. If $j$ is large enough, then we have

$$
\left|\bar{X}_{j}-\bar{Y}_{j}\right|<r_{2}
$$

and

$$
\left|Z_{j}-Y_{j}\right| \leq\left|Z_{j}-Z\right|+\left|Z-Y_{j}\right|<r+\frac{r_{2}}{2}<r_{2} .
$$

By (2.8) we obtain

$$
\left|\eta\left(Z_{j}\right)-\eta_{j}\left(\bar{X}_{j}\right)\right| \leq C_{2}\left|Z_{j}-\bar{X}_{j}\right|^{\alpha}
$$

Since $\lim _{j \rightarrow \infty} z_{j}^{1}=1$, and $\left|z_{j}^{2}\right|<(\tan \alpha-\varepsilon) z_{j}^{1}$, so by Theorem 3 of [6], for any subsequence $\bar{Z}_{j}$ of $Z_{j}$, we can always find a subsequence $\bar{Z}_{j}^{\prime}$ of $\bar{Z}_{j}$ such that $\lim _{j \rightarrow \infty} \eta_{j}\left(\bar{Z}_{j}^{\prime}\right)=(-1,0,0)$.

Therefore $\lim _{j \rightarrow \infty} \eta_{j}\left(Z_{j}\right)=(-1,0,0)=\eta$.

Also, it is easy to see from (2.9) that

$$
\limsup _{j \rightarrow \infty}\left|Z_{j}-\bar{X}_{j}\right| \leq r
$$

Let $j \rightarrow \infty$ in (2.10), we then have

$$
\limsup _{j \rightarrow \infty}\left|\eta-\eta_{j}\left(\bar{X}_{j}\right)\right| \leq C_{2} r^{\alpha} \text {. }
$$

Now let $r \rightarrow 0$, we conclude that $\lim _{j \rightarrow \infty}\left|\eta-\eta_{j}\left(\bar{X}_{j}\right)\right|=0$. The proof of Theorem 2.2 is then completed.

Combining Theorems 2.1 and 2.2, we get

THEOREM. The unit normal vector $\left(T u,-1 / \sqrt{1+|D u|^{2}}\right)$ extends to be continuous on $\bar{\Omega}$. More precisely,

$$
\lim _{\substack{x \rightarrow 0 \\ x \in \bar{\Omega}-\{0\}}}\left(T u(x), \frac{-1}{\sqrt{1+|D u(x)|^{2}}}\right)=(-1,0,0) .
$$

Acknowledgment. I wish to thank Robert Finn and Brian White for useful discussions.

\section{REFERENCES}

[1] P. Concus and R. Finn, Capillary free surfaces in a gravitational field, Acta Math., 132 (1974), 207-223.

[2] R. Finn, Existence criteria for capillary free surfaces without gravity, Indiana Univ. Math. J., 32 (1983), 439-460. 
[3] E. Giusti, Generalized solutions of mean curvature equations, Pacific J. Math., 88 (1980), 297-321.

[4] Minimal surfaces and functions of bounded variation. Notes on pure mathematics. Australian National Univ., Canberra (1977).

[5] N. J. Korevaar, On the behavior of a capillary surface at a re-entrant corner, Pacific J. Math., 88 (1980), 379-385.

[6] U. Massari and L. Pepe, Sulle successioni convergenti di superfici a curvatura media assegnata, Rend. Sem. Mat. Padova, 53 (1975), 53-68.

[7] M. Miranda, Un principio di massimo forte per le frontiere minimali ecc., Rend. Sem. Mat. Padova, 45 (1971), 355-366.

[8] _ Superfici minime illimitate, Ann. Scuola Norm. Sup. Pisa, (4) 4 (1977), 313-322.

[9] L. Simon, Regularity of capillary surfaces over domains with corners, Pacific J. Math., 88 (1980), 363-377.

[10] L.-F. Tam. The behavior of capillary surfaces as gravity tends to zero, to appear in Comm. in Partial Differential Equations.

[11] Existence criteria for capillary free surfaces without gravity, to appear in Pacific J. Math.

[12] J. Taylor, Boundary regularity for solutions to various capillarity and free boundary problems, Comm. in Partial Differential Equations, 2 (1977), 323-357.

Received February 7, 1984.

Purdue UnIVERSITY

WEST LAFA YETTE, IN 47907 


\section{PACIFIC JOURNAL OF MATHEMATICS EDITORS}

\author{
V. S. VARADARAJAN \\ (Managing Editor) \\ University of California \\ Los Angeles, CA 90024 \\ Herbert Clemens \\ University of Utah \\ Salt Lake City, UT 84112 \\ R. FINN \\ Stanford University \\ Stanford, CA 94305
}

\author{
HERMANN FLASCHKA \\ University of Arizona \\ Tucson, AZ 85721 \\ RAMESH A. GANGOLLI \\ University of Washington \\ Seattle, WA 98195 \\ VAUGHAN F. R. JONES \\ University of California \\ Berkeley, CA 94720 \\ ROBION KIRBY \\ University of California \\ Berkeley, CA 94720
}

C. C. MOORE

University of California

Berkeley, CA 94720

H. SAMELSON

Stanford University

Stanford, CA 94305

HAROLD STARK

University of California, San Diego La Jolla, CA 92093

\section{ASSOCIATE EDITORS}
R. ARENS
E. F. BECKENBACH (1906-1982)
B. H. NEUMANN
F. WOLF
K. YosHIDA

\section{SUPPORTING INSTITUTIONS}

\begin{abstract}
UNIVERSITY OF ARIZONA
UNIVERSITY OF BRITISH COLUMBIA

CALIFORNIA INSTITUTE OF TECHNOLOGY

UNIVERSITY OF CALIFORNIA

MONTANA STATE UNIVERSITY

UNIVERSITY OF NEVADA, RENO

NEW MEXICO STATE UNIVERSITY OREGON STATE UNIVERSITY
\end{abstract}

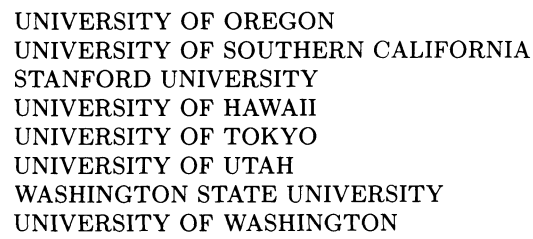

The Supporting Institutions listed above contribute to the cost of publication of this Journal, but they are not owners or publishers and have no responsibility for its content or policies.

Mathematical papers intended for publication in the Pacific Journal of Mathematics should be in typed form or offset-reproduced (not dittoed), double spaced with large margins. Please do not use built up fractions in the text of the manuscript. However, you may use them in the displayed equations. Underline Greek letters in red, German in green, and script in blue. The first paragraph must be capable of being used separately as a synopsis of the entire paper. In particular it should contain no bibliographic references. Please propose a heading for the odd numbered pages of less than 35 characters. Manuscripts, in triplicate, may be sent to any one of the editors. Please classify according to the scheme of Math. Reviews, Index to Vol. 39. Supply name and address of author to whom proofs should be sent. All other communications should be addressed to the managing editor, or Elaine Barth, University of California, Los Angeles, California 90024.

There are page-charges associated with articles appearing in the Pacific Journal of Mathematics. These charges are expected to be paid by the author's University, Government Agency or Company. If the author or authors do not have access to such Institutional support these charges are waived. Single authors will receive 50 free reprints; joint authors will receive a total of 100 free reprints. Additional copies may be obtained at cost in multiples of 50 .

The Pacific Journal of Mathematics is issued monthly as of January 1966. Regular subscription rate: $\$ 190.00$ a year (5 Vols., 10 issues). Special rate: $\$ 95.00$ a year to individual members of supporting institutions.

Subscriptions, orders for numbers issued in the last three calendar years, and changes of address should be sent to Pacific Journal of Mathematics, P.O. Box 969, Carmel Valley, CA 93924, U.S.A. Old back numbers obtainable from Kraus Periodicals Co., Route 100, Millwood, NY 10546.

The Pacific Journal of Mathematics at P.O. Box 969, Carmel Valley, CA 93924 (ISSN 0030-8730) publishes 5 volumes per year. Application to mail at Second-class postage rates is pending at Carmel Valley, California, and additional mailing offices. Postmaster: send address changes to Pacific Journal of Mathematics, P.O. Box 969, Carmel Valley, CA 93924.

PUBLISHED BY PACIFIC JOURNAL OF MATHEMATICS, A NON-PROFIT CORPORATION Copyright (C) 1986 by Pacific Journal of Mathematics 


\section{Pacific Journal of Mathematics}

Vol. 124, No. $2 \quad$ June, 1986

Philip Lee Bowers, Nonshrinkable "cell-like" decompositions of $s \ldots \ldots .257$

Aurelio Carboni and Ross Street, Order ideals in categories .......... 275

Leoni Dalla, Increasing paths on the one-skeleton of a convex compact set in

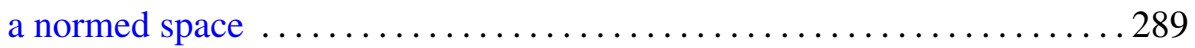

Jim Hoste, A polynomial invariant of knots and links ............... 295

Sheldon Katz, Tangents to a multiple plane curve ................... 321

Thomas George Lucas, Some results on Prüfer rings $\ldots \ldots \ldots \ldots \ldots \ldots 33$

Pham Anh Minh, Modular invariant theory and cohomology algebras of

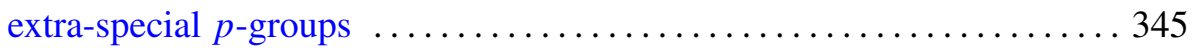

Ikuko Miyamoto, On inclusion relations for absolute Nörlund

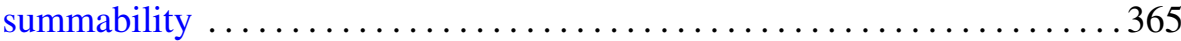

A. Papadopoulos, Geometric intersection functions and Hamiltonian flows on the space of measured foliations on a surface ............. 375

Richard Dean Resco, J. Toby Stafford and Robert Breckenridge

Warfield, Jr., Fully bounded $G$-rings $\ldots \ldots \ldots \ldots \ldots \ldots \ldots \ldots \ldots 4$

Haskell Paul Rosenthal, Functional Hilbertian sums .................417

Luen-Fai Tam, Regularity of capillary surfaces over domains with corners: borderline case ................................. 469

Hugh C. Williams, The spacing of the minima in certain cubic lattices .....483 